\title{
Genetic association between the rs 12252 SNP of the interferon-induced transmembrane protein gene and influenza A virus infection in the Korean population
}

\author{
Yong-Chan Kim ${ }^{1,2} \cdot$ Min-Ju Jeong ${ }^{1,2} \cdot$ Byung-Hoon Jeong ${ }^{1,2}(\mathbb{D}$
}

Accepted: 21 October 2020 / Published online: 4 November 2020

(c) The Korean Society of Toxicogenomics and Toxicoproteomics 20202020

\begin{abstract}
Background Interferon-induced transmembrane protein 3 (IFITM3) is a potent host antiviral effector protein that blocks the invasion of various viruses, including the influenza A virus (IAV). The $\mathrm{C}$ allele of the rs 12252 single nucleotide polymorphism (SNP) shows vulnerability to the pandemic 2009 H1N1 IAV in European and Asian populations.

Objective Here, we estimated the disease susceptibility of the rs12252 SNP with the pandemic 2009 H1N1 IAV infection in the Korean population.

Results We carried out direct sequencing of the IFITM3 gene and compared the genotype and allele frequencies of the rs 12252 SNP of the IFITM3 gene in healthy Koreans and pandemic 2009 H1N1 IAV-infected patients. Notably, we observed that healthy individuals had a similar genotype distribution of the rs12252 SNP $(P=0.140)$ as patients. The dominant model and recessive model did not find a statistically significant difference in genotype distribution between healthy individuals and patients. In addition, the allele distribution of the rs 12252 SNP of in healthy individuals and patients also showed a similar genetic distribution $(P=0.757)$. However, the genetic distribution of rs 12252 SNP in merged patient group (Koreans and Chinese populations) showed significant association with susceptibility of pandemic $2009 \operatorname{IAV}(P=0.0393)$.

Conclusion To the best of our knowledge, this was the first evaluation of the susceptibility of the pandemic 2009 H1N1 IAV in the Korean population.
\end{abstract}

Keywords IFITM3 $\cdot$ Single nucleotide polymorphism $\cdot$ rs12252 SNP $\cdot$ Case-control study

\section{Introduction}

Interferon-induced transmembrane protein 3 (IFITM3) is a host antiviral effector protein that augments expression levels by type I and II interferons to respond to the invasion of various viruses, including influenza A virus (IAV) (Brass et al. 2009; Weidner et al. 2010; Bailey et al. 2012; Diamond

Yong-Chan Kim and Min-Ju Jeong contributed equally to this work.

Byung-Hoon Jeong bhjeong@jbnu.ac.kr

1 Korea Zoonosis Research Institute, Jeonbuk National University, 820-120, Hana-ro, Iksan, Jeonbuk 54531, Republic of Korea

2 Department of Bioactive Material Sciences, Jeonbuk National University, Jeonju, Jeonbuk 54896, Republic of Korea and Farzan 2013; Kim et al. 2019; Lee et al. 2019). IFITM3 protein is localized in late endosomes and inhibits endosomal escape of influenza A viruses (Feeley et al. 2011). In particular, the $\mathrm{N}$-terminal domain of the IFITM3 protein contains a sorting signal motif and plays a pivotal role in correct localization to the endosome and in blocking viral infections (Jia et al. 2012, 2014; Li et al. 2013).

Recent studies showed that single nucleotide polymorphisms (SNPs) of the IFITM3 gene influence the antiviral capacity of the IFITM3 protein and are associated with the severity of the pandemic 2009 H1N1 IAV, which had a disastrous effect worldwide (van 't Klooster et al. 2010; Tramuto et al. 2011; Kim 2016). Among these SNPs, the $\mathrm{C}$ allele of the rs12252 SNP, which is located on the splicing receptor site, makes a 21 amino acid-shortened splicing isoform of the IFITM3 protein and shows susceptibility to the pandemic 2009 H1N1 IAV (Everitt et al. 2012). According to the 1000 genome database, although the European population has only $0.3 \%$ of the rs 12252 SNP CC genotype, 
the CC genotype was found in 5.7\% of hospitalized patients and showed a statistically significant association with the susceptibility to the pandemic 2009 H1N1 IAV. The correlation of the rs12252 SNP with the susceptibility to the severe pandemic 2009 H1N1 IAV was reaffirmed in Han Chinese populations, and there was a strong association between the number of intensive care unit patients and the rs 12252 SNP CC genotype (Zhang et al. 2013; Lee et al. 2017). In addition, a case-control study in the Caucasian population identified an association of the rs 12252 SNP with mild influenza infection (Mills et al. 2014). Previous study investigated the number of deaths from the pandemic 2009 H1N1 IAV and tried to find a correlation between the disease severity of influenza A virus infection and the rs12252 SNP; however, the study did not find an association of the rs12252 SNP with influenza severity (Kim and Jeong 2017). Although the relationship between influenza severity and rs12252 SNP was elusive (Lopez-Rodriguez et al. 2016), a cross-ethnic case-control study and a meta-analysis have validated the disease association of the rs 12252 SNP with the pandemic 2009 H1N1 IAV (Xuan et al. 2015; Yang et al. 2015; Chen et al. 2018; Makvandi-Nejad et al. 2018; Prabhu et al. 2018). Several studies on ethnic groups have confirmed the relationship between the rs12252 SNP and the susceptibility to the pandemic 2009 H1N1 IAV, but an evaluation of the susceptibility to this virus in the Korean population has not been performed thus far.

In the present study, we estimated the disease susceptibility of the rs 12252 SNP with the pandemic 2009 H1N1 IAV infection in the Korean population. For this, we carried out direct sequencing of the IFITM3 gene and analyzed the genotype and allele frequencies of the rs 12252 SNP of the IFITM 3 gene between healthy individuals and pandemic 2009 H1N1 IAV-infected patients in Korea.

\section{Materials and methods}

\section{Subjects}

Thirty blood samples of laboratory-confirmed pandemic 2009 H1N1 IAV-infected patients were provided from the Jeonbuk National University Hospital Biobank, a member of the Korea Biobank Network. A total of 204 blood samples from healthy Korean subjects were obtained from the Korea Biobank Network at the Centers for Disease Control and Prevention. All samples derived from the Korea Biobank Network were obtained with informed consent under institutional review board-approved protocols. The exclusion criteria of healthy Koreans included diabetes, high blood pressure, gastritis, gastric ulcer, myocardial infarction, thyroid disease, congestive heart failure, coronary artery disease, hypothyroidism asthma, chronic lung disease, peripheral vascular disease, kidney disease, hepatitis, tuberculosis, cerebrovascular disease, head trauma, urinary tract infection, arthritis and cancer. All the samples and related data were anonymized prior to the analysis.

\section{Genomic DNA extraction}

Genomic DNA was extracted from $200 \mu$ l of blood using the Blood Genomic DNA Isolation Kit (Qiagen, Valencia, California, USA) following the manufacturer's instructions.

\section{Amplification of the IFITM3 gene and genetic analysis}

The human IFITM3 gene was amplified from genomic DNA using forward and reverse gene-specific primers. The sequences of the primers were as follows: IFITM3-F (5'CAGGGGAAGTCTCCAGGACC-3') and IFITM3-R (5'CCAAGCCACACACACACACA-3'). Polymerase chain reaction (PCR) was performed using GoTaq ${ }^{\circledR}$ DNA Polymerase (Promega, Fitchburg, Wisconsin, USA). The PCR mixture contained 20 pmol of each primer, $5 \mu$ l of $10 \times$ Taq DNA polymerase buffer, $1 \mu \mathrm{l}$ of $10 \mathrm{mM}$ dNTP mixture and 2.5 units of Taq DNA polymerase.

The PCR conditions of IFITM3-F and IFITM3-R primers were $94^{\circ} \mathrm{C}$ for $2 \mathrm{~min}$ to denature; 35 cycles of $94^{\circ} \mathrm{C}$ for $45 \mathrm{~s}$, $71{ }^{\circ} \mathrm{C}$ for $45 \mathrm{~s}$, and $72{ }^{\circ} \mathrm{C}$ for $1 \mathrm{~min} 30 \mathrm{~s}$; and then 1 cycle of $72{ }^{\circ} \mathrm{C}$ for $10 \mathrm{~min}$ to extend the reaction. PCR was performed using S-1000 Thermal Cycler (Bio-Rad, Hercules, California, USA). The PCR products were purified using the PCR Purification Kit (Thermo Fisher Scientific, Bridgewater, New Jersey, USA) and directly sequenced with an ABI 3730 Automated Sequencer (ABI, Foster City, California, USA). Sequencing results were read by Finch TV software (Geospiza Inc, Seattle, Washington, USA), and genotyping was carried out.

\section{Literature search}

A literature search was conducted to looking for rs 12252 SNP of the IFITM3 gene in previous studies. The searching terms were: "IFITM3", "SNP", "IAV" combined with "pandemic" or "susceptibility". Moreover, we supplemented our search by screening the reference lists of the relevant studies, including the original article. References for all identified publications were indicated in Table 2 .

\section{Genetic analysis}

Statistical analyses were performed using SAS version 9.4 (SAS Institute Inc., USA). The differences in genotype and allele frequencies of the IFITM 3 gene between case and control populations compared using $\chi^{2}$ test. The 
Table 1 The detailed information of the study population

\begin{tabular}{lll}
\hline Characteristics & Cases & Controls \\
\hline Number & 30 & 204 \\
Age & $55.27 \pm 17.88$ & $62.43 \pm 8.96$ \\
Sex $(n, \%)$ & & \\
Male & $11(36.67)$ & $74(36.27)$ \\
Female & $19(63.33)$ & $130(63.73)$ \\
\hline
\end{tabular}

Hardy-Weinberg Equilibrium (HWE) test was performed using HWE calculator (https://www.genes.org.uk/software/ hardy-weinberg.shtml).

\section{Results}

\section{Subject description}

A total of 234 individuals were included in the association analysis. Detailed information on the study population is described in Table 1. A total of 30 pandemic 2009 H1N1 IAV-diagnosed patients were composed of 19 females and 11 males. A total of 204 healthy individuals were composed of 130 females and 74 males. The mean age at diagnosis of $2009 \mathrm{H} 1 \mathrm{~N} 1 \mathrm{IAV}$-infected patients was $55.27 \pm 17.88$ years, and the mean age of healthy individuals at sample collection was $62.43 \pm 8.96$ years.

\section{Genotyping and HWE analyses}

We performed direct sequencing in 204 healthy individuals and 30 pandemic 2009 H1N1 IAV-infected patients and carried out genotyping and HWE analyses. Detailed information on the genotyping results and HWE values in the Korean population is described in Table 2.

\section{Evaluation of susceptibility of H1N1 influenza 2009 pandemic virus infection in the Korean population}

To estimate disease the susceptibility of the rs12252 SNP of the IFITM3 gene with the pandemic 2009 H1N1 IAV in the Korean population, we performed a case-control association study. We observed that healthy individuals had a similar genotype distribution of the rs12252 SNP $(P=0.1403)$ to patients in the Korean population. Among 16 groups investigated in previous studies, the healthy Han Chinese population has a most similar distribution of rs12252 SNP with the healthy Korean population in genotype $(P=0.4442)$ and allele $(P=0.2119)$ frequencies (Table 2$)$. We additionally tried to analyze association using the dominant model and recessive model in the Korean population.

\section{Analyses in dominant and recessive models}

To find risk factor on the susceptibility of pandemic 2009 H1N1 IAV infection in Korean population, two genetic models, including dominant and recessive models were performed in this study. In dominant model, the distribution of $\mathrm{CC}+\mathrm{CT}$ and TT genotypes is $163(79.9 \%)$ and $41(20.1 \%)$ in control and $28(93.3 \%)$ and $2(6.7 \%)$ in case, respectively. The frequency of the CC + CT genotypes in pandemic 2009 $\mathrm{H} 1 \mathrm{~N} 1 \mathrm{IAV}$-infected patients is substantially greater than that in the normal Korean population. However, the dominant model $(P=0.076)$ and the recessive model $(P=0.757)$ did not find a statistically significant difference in the genotype distribution between healthy individuals and patients (Table 3). In addition, the allele distribution of the rs 12252 SNP in healthy individuals and patients also showed a similar genetic distribution $(P=0.437$, Table 2$)$.

\section{Discussion}

In the present study, we estimated the susceptibility of the rs12252 SNP to the pandemic 2009 H1N1 IAV in the Korean population. Interestingly, all genetic models performed in this study showed no correlation between the rs12252 SNP and the susceptibility of the pandemic 2009 H1N1 IAV in the Korean population. Further research is needed to assess whether this result is due to ethnic background. In addition, because it can be triggered by the small sample size of pandemic 2009 H1N1 IAV-infected patients in the Korean population, further study in a large population should be performed to validate the correlation between the rs 12252 SNP and the susceptibility of the pandemic 2009 H1N1 IAV in the Korean population. However, there are severe limitations in this study due to small sample numbers of pandemic 2009 H1N1 IAV-infected patients and the impossibility of stratified study according to disease severity under current status of Korea biobank. Sample collection is systematically needed at the national level for preemptive control of pandemic diseases. Indeed, the mechanism of the antiviral capacity of the rs 12252 SNP is elusive. Previous in vivo and in vitro studies failed to detect the 21 amino acid-shortened splicing isoform of the IFITM3 protein induced by the rs12252 SNP C allele (Makvandi-Nejad et al. 2018).

In recent a study, the rs34481144 SNP, which is located on the promoter of the IFITM3 gene, showed an association with the severity of the pandemic 2009 H1N1 IAV (Allen et al. 2017; David et al. 2018). The rs34481144 SNP potently influenced the host innate immune system by modulating not only the expression level of the IFITM3 


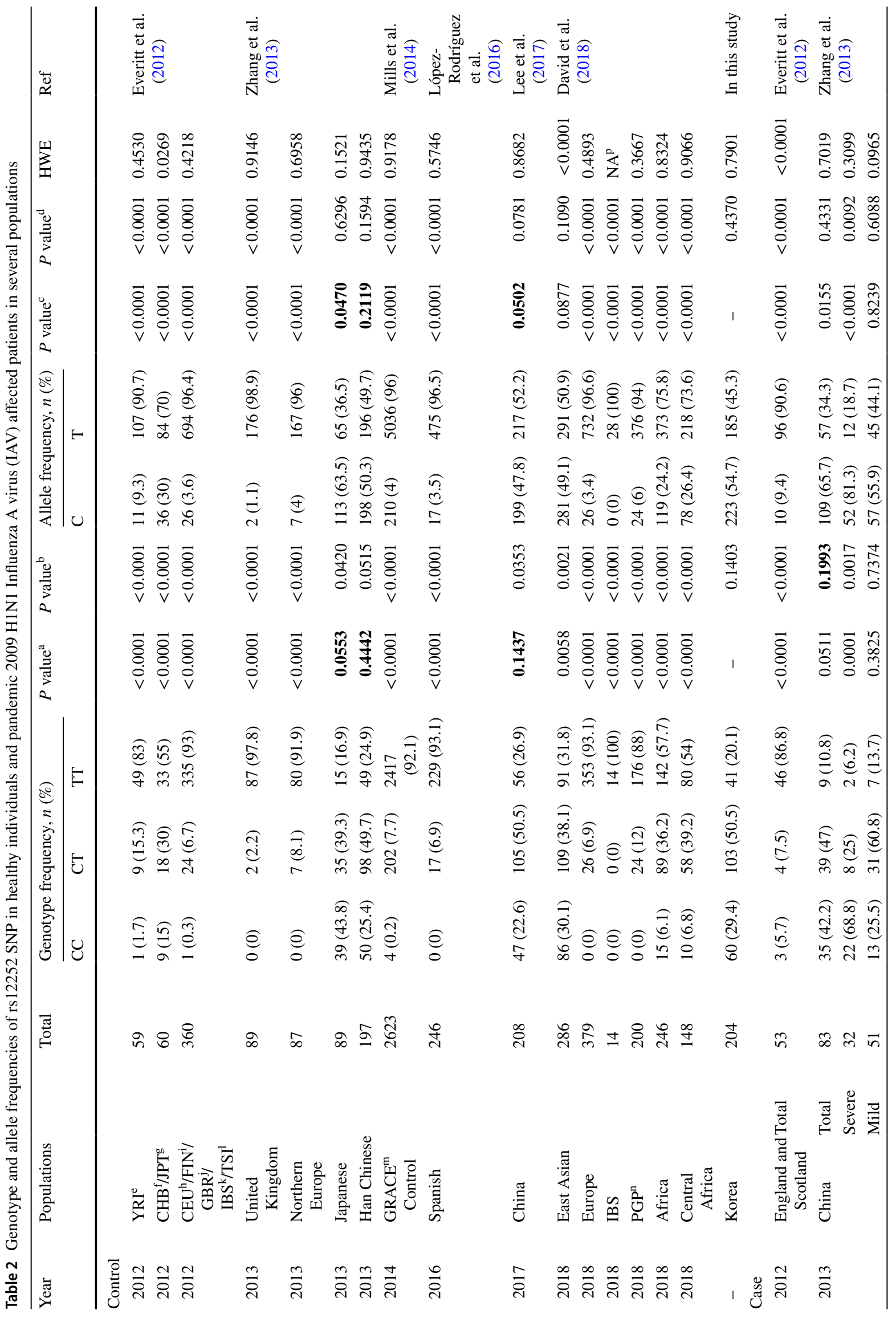




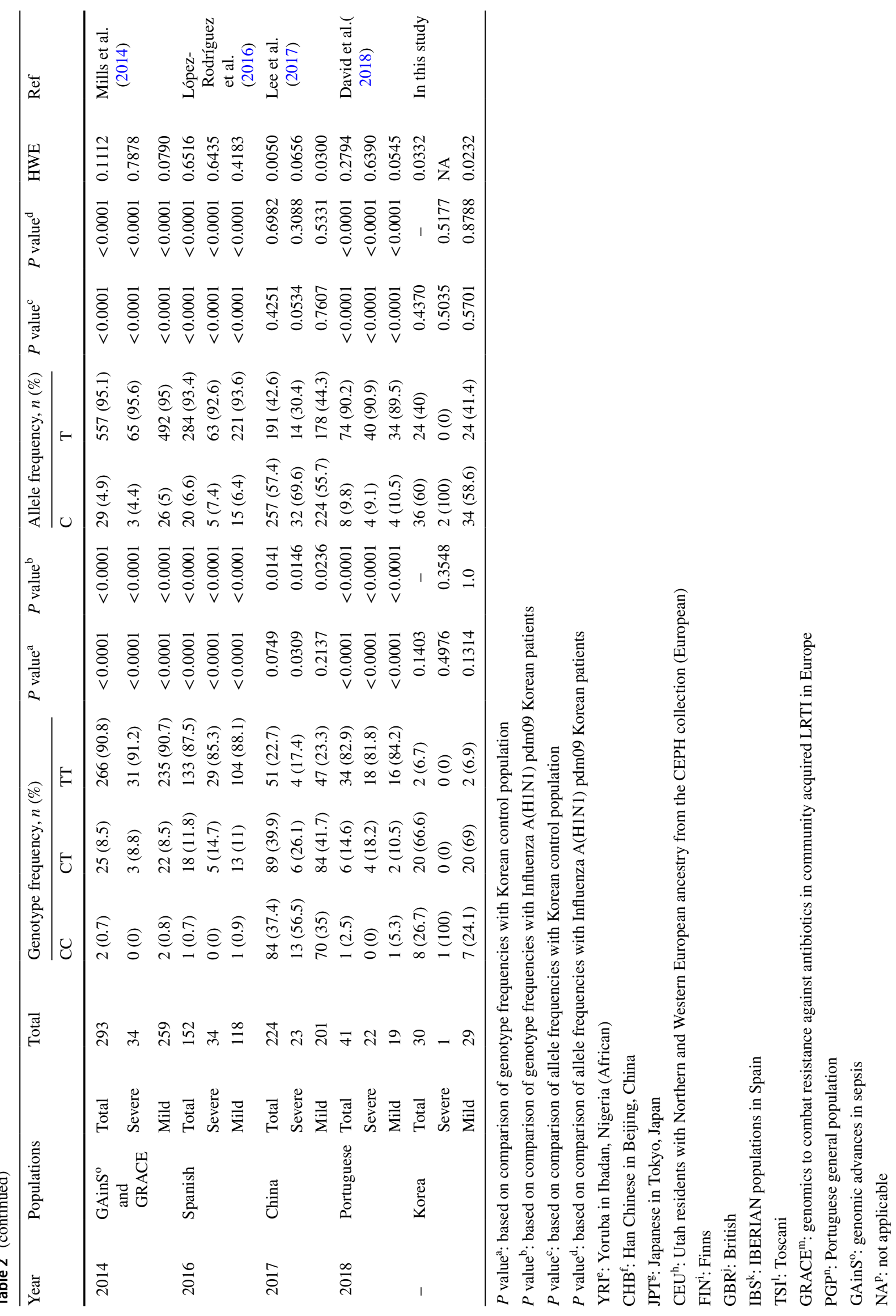


Table 3 Association analysis in the dominant and recessive models in Korean population

\begin{tabular}{llll}
\hline Genetic model & Control & Case & $P$ value \\
\hline $\begin{array}{l}\text { Dominant model, } n(\%) \\
\text { CC }+ \text { CT }\end{array}$ & $163(79.9)$ & $28(93.3)$ & 0.076 \\
TT & $41(20.1)$ & $2(6.7)$ & \\
Recessive model, $n(\%)$ & & & \\
CC & $60(29.4)$ & $8(26.7)$ & 0.757 \\
CT + TT & $144(70.6)$ & $22(73.3)$ & \\
Total, $n$ & 204 & 30 & \\
\hline
\end{tabular}

gene but also those of neighboring genes (Allen et al. 2017). In addition, the rs6598045 SNP was associated with the binding ability of the transcription factor of the IFITM3 gene and related to the susceptibility of the pandemic 2009 H1N1 IAV. T allele of rs6598045 SNP which is more prevalent in 2009 pandemic influenza-infected patients showed reduced promoter activity compared to C allele of rs6598045 which is more prevalent in healthy control (Kim et al. 2020). Because of the close genetic locus among the three SNPs, including rs12252 SNP, rs34481144 SNP and rs6598045 SNP, investigation of the relationship among them is highly desirable in the future.

In conclusion, we investigated the genotype and allele frequencies of the rs12252 SNP of the IFITM3 gene and estimated the susceptibility of the pandemic 2009 H1N1 IAV in the Korean population. We found no correlation between the genotype, allele and dominant and recessive models of the rs 12252 SNP and the vulnerability of the pandemic 2009 H1N1 IAV in the Korean population. To the best of our knowledge, this was the first evaluation of the susceptibility of the pandemic 2009 H1N1 IAV in the Korean population.

Acknowledgements The biospecimens and data used in this study were provided by the Biobank of Jeonbuk National University Hospital, a member of the Korea Biobank Network, which is supported by the Ministry of Health, Welfare and Family Affairs. All samples derived from the Korea Biobank Network were obtained with informed consent under institutional review board-approved protocols. This research was supported by the Basic Science Research Program through the National Research Foundation of Korea (NRF) funded by the Ministry of Education (2017R1A6A1A03015876). This research was supported by the Basic Science Program through the National Research Foundation of Korea (NRF) funded by the Ministry of Education, Science and Technology (2018R1D1A1B07048711). This research was supported by "Research Base Construction Fund Support Program" funded by Jeonbuk National University in 2020. Min-Ju Jeong was supported by the BK21 Plus Program in the Department of Bioactive Material Sciences. This work was supported by NRF (National Research Foundation of Korea) Grant funded by the Korean Government (NRF-2019-Fostering Core Leaders of the Future Basic Science Program/Global Ph.D. Fellowship Program). This work was supported by Basic Science Research Program through the National Research Foundation of Korea (NRF) funded by the Ministry of Education (2020R1A6A3A1307432311). The funders had no role in study design, data collection and analysis, decision to publish, or preparation of the manuscript.
Author contributions Y.C. Kim, M.J. Jeong and B.H. Jeong conceived and designed the experiments. Y.C. Kim and M.J. Jeong performed the experiments. Y.C. Kim and B.H. Jeong analyzed the data. Y.C. Kim, M.J. Jeong and B.H. Jeong wrote the paper. All authors read and approved the final manuscript.

\section{Compliance with ethical standards}

Conflict of interest The authors declare no conflict of interest.

Ethical approval All procedures performed in the present study were accredited by the institutional review board of the Jeonbuk National University and were in accordance with the 1964 Helsinki Declaration and its later amendments or comparable ethical standards (Approval number: JBNU 2017-08-009).

\section{References}

Allen EK et al (2017) SNP-mediated disruption of CTCF binding at the IFITM3 promoter is associated with risk of severe influenza in humans. Nat Med 23(8):975-983

Bailey CC, Huang IC, Kam C, Farzan M (2012) Ifitm3 limits the severity of acute influenza in mice. PLoS Pathog 8(9):e1002909

Brass AL et al (2009) The IFITM proteins mediate cellular resistance to influenza A H1N1 virus, West Nile virus, and dengue virus. Cell 139(7):1243-1254

Chen $\mathrm{T}$ et al (2018) Association between rs12252 and influenza susceptibility and severity: an updated meta-analysis. Epidemiol Infect 147:1-9. https://doi.org/10.1017/S0950268818002832

David S et al (2018) Population genetics of IFITM3 in Portugal and Central Africa reveals a potential modifier of influenza severity. Immunogenetics 70(3):169-177

Diamond MS, Farzan M (2013) The broad-spectrum antiviral functions of IFIT and IFITM proteins. Nat Rev Immunol 13(1):46-57

Everitt AR et al (2012) IFITM3 restricts the morbidity and mortality associated with influenza. Nature 484(7395):519-523

Feeley EM et al (2011) IFITM3 inhibits influenza A virus infection by preventing cytosolic entry. PLoS Pathog 7(10):e1002337

Jia $R$ et al (2012) The N-terminal region of IFITM3 modulates its antiviral activity by regulating IFITM3 cellular localization. J Virol 86(24):13697-13707

Jia R et al (2014) Identification of an endocytic signal essential for the antiviral action of IFITM3. Cell Microbiol 16(7):1080-1093

Kim JY (2016) The 2009 H1N1 Pandemic Influenza in Korea. Tuberc Respir Dis (Seoul) 79(2):70-73

Kim YC, Jeong BH (2017) No correlation of the disease severity of influenza A virus infection with the rs 12252 polymorphism of the 
interferon-induced transmembrane protein 3 gene. Intervirology 60(1-2):69-74

Kim YC, Jeong MJ, Jeong BH (2019) Genetic characteristics and polymorphisms in the chicken interferon-induced transmembrane protein (IFITM3) gene. Vet Res Commun 43(4):203-214

Kim YC, Jeong MJ, Jeong BH (2020) Strong association of regulatory single nucleotide polymorphisms (SNPs) of the IFITM3 gene with influenza H1N1 2009 pandemic virus infection. Cell Mol Immunol 17(6):662-664

Lee N et al (2017) IFITM3, TLR3, and CD55 gene SNPs and cumulative genetic risks for severe outcomes in Chinese patients with H7N9/H1N1pdm09 influenza. J Infect Dis 216(1):97-104

Lee YJ, Kang JH, Yun MH, Lee SB (2019) Curcumin inhibits poly (dA:dT)-induced IL-18 secretion by inhibiting the ASC oligomerization in human keratinocytes. Mol Cell Toxicol 15(4):399-406

Li K et al (2013) IFITM proteins restrict viral membrane hemifusion. PLoS Pathog 9(1):e1003124

Lopez-Rodriguez M et al (2016) IFITM3 and severe influenza virus infection. No evidence of genetic association. Eur J Clin Microbiol Infect Dis Off Publ Eur Soc Clin Microbiol 35(11):1811-1817

Makvandi-Nejad S et al (2018) Lack of Truncated IFITM3 transcripts in cells homozygous for the rs $12252-\mathrm{C}$ variant that is associated with severe influenza infection. J Infect Dis 217(2):257-262

Mills TC et al (2014) IFITM3 and susceptibility to respiratory viral infections in the community. J Infect Dis 209(7):1028-1031

Prabhu SS, Chakraborty TT, Kumar N, Banerjee I (2018) Association between IFITM3 rs12252 polymorphism and influenza susceptibility and severity: a meta-analysis. Gene 674(20):70-79
Tramuto F et al (2011) Surveillance of hospitalised patients with influenza-like illness during pandemic influenza $\mathrm{A}(\mathrm{H} 1 \mathrm{~N} 1)$ season in Sicily, April 2009-December 2010. Euro Surveill 16:35

van 't Klooster TM et al (2010) Surveillance of hospitalisations for 2009 pandemic influenza A(H1N1) in the Netherlands, 5 June-31 December 2009. Euro Surveill 15:2

Weidner JM et al (2010) Interferon-induced cell membrane proteins, IFITM3 and tetherin, inhibit vesicular stomatitis virus infection via distinct mechanisms. J Virol 84(24):12646-12657

Xuan $Y$ et al (2015) IFITM3 rs12252 T $>$ C polymorphism is associated with the risk of severe influenza: a meta-analysis. Epidemiol Infect 143(14):2975-2984

Yang X et al (2015) Interferon-inducible transmembrane protein 3 genetic variant rs 12252 and influenza susceptibility and severity: a meta-analysis. PLoS ONE 10(5):e0124985

Zhang YH et al (2013) Interferon-induced transmembrane protein-3 genetic variant rs12252-C is associated with severe influenza in Chinese individuals. Nat Commun 4:1418

Publisher's Note Springer Nature remains neutral with regard to jurisdictional claims in published maps and institutional affiliations. 